\title{
A Scheme for Calculating Atomic Structures beyond the Spherical Approximation
}

\author{
Mitiyasu Miyasita ${ }^{1}$, Katsuhiko Higuchi ${ }^{2}$, Masahiko Higuchi ${ }^{3}$ \\ ${ }^{1}$ Graduate School of Science and Engineering, Shinshu University, Ueda, Japan \\ ${ }^{2}$ Graduate School of Advanced Science of Matter, Hiroshima University, Higashi-Hiroshima, Japan \\ ${ }^{3}$ Department of Physics, Faculty of Science, Shinshu University, Matsumoto, Japan \\ E-mail: miyasita.mitiyasu@gmail.com \\ Received February 11, 2011; revised April 3, 2011; April 4, 2011
}

\begin{abstract}
We present a scheme for calculating atomic single-particle wave functions and spectra with taking into account the nonspherical effect explicitly. The actual calculation is also performed for the neutral carbon atom within the Hartree-Fock-Slater approximation. As compared with the conventional atomic structure of the spherical approximation, the degenerate energy levels are split partially. The ground state values of the total orbital and spin angular momenta are estimated to be both about unity, which corresponds to the term ${ }^{3} \mathrm{P}$ in the LS-multiplet theory. This means that the nonspherical effect may play an essential role in the description of the magnetization caused by the orbital polarization.
\end{abstract}

Keywords: Nonspherical Distribution of Electrons, Spherical Approximation, Orbital Polarization, Atomic Structure, Carbon Atom

\section{Introduction}

Let us start with revisiting the conventional atomic structures. We consider the isolated neutral atom with the atomic number $Z$. Neglecting the relativistic effects, the Schrödinger Equation for the stationary state is given by

$$
\hat{H} \Psi=E \Psi
$$

with

$$
\hat{H}=\sum_{i=1}^{Z}\left(-\nabla_{i}^{2}-\frac{2 Z}{r_{i}}\right)+\frac{1}{2} \sum_{\substack{i, j \\(i \neq j)}}^{Z} \frac{2}{\left|\boldsymbol{r}_{i}-\boldsymbol{r}_{j}\right|},
$$

where $r_{i}$ and $r_{i}$ stand for the position of the ith electron and its magnitude, respectively, and where the atomic unit is used. Equation (1) can be numerically solved only in small atomic systems, but in larger atomic systems we have to utilize the theories to reduce Equation (1) into the effective single-particle Equation such as the Hartree [1], Hartre-Fock [2] and Kohn-Sham [3,4] Equations, etc. The single-particle Equation is generally written by

$$
\left\{-\nabla^{2}+V_{i \sigma}(\boldsymbol{r})\right\} \psi_{i \sigma}(\boldsymbol{r})=\varepsilon_{i \sigma} \psi_{i \sigma}(\boldsymbol{r}),
$$

where $\sigma$ denotes the up-spin $\alpha$ or down-spin $\beta$. In order to solve Equation (3), we have usually used the spherical approximation, i.e., the central field approximation [5]. Under such the approximation, Equation (3) is separable into two Equations, one of which depends on the radial variable $r$ and the other on the angular variables, $\theta$ and $\varphi$. If the effective potential is spherically symmetric and local [6], and if the solutions are given by

$$
\phi_{n l m \sigma}(\boldsymbol{r})=\frac{1}{r} p_{n l}^{\sigma}(r) Y_{l m}(\theta, \varphi),
$$

then two Equations are

$$
\begin{gathered}
\left\{-\frac{\mathrm{d}^{2}}{\mathrm{~d} r^{2}}+\frac{l(l+1)}{r^{2}}+V_{\sigma}(r)\right\} p_{n l}^{\sigma}(r)=\varepsilon_{n l \sigma}^{0} p_{n l}^{\sigma}(r), \\
\hat{\boldsymbol{I}}^{2} Y_{l m}(\theta, \varphi)=l(l+1) Y_{l m}(\theta, \varphi),
\end{gathered}
$$

where $\hat{\boldsymbol{l}}$ is the operator of the orbital angular momentum, and $Y_{l m}(\theta, \varphi)$ are the spherical harmonics [7]. Radial wave functions $p_{n l}^{\sigma}(r)$ of Equation (5) are calculated easily by means of the numerical methods such as the Herman-Skillman method [8,9]. Thus, the conventional atomic structures, where the eigenstates are specified by the quantum numbers $n, l(\leq n-1), m(-l \leq m \leq l)$ and $\sigma$ can be obtained.

Here the question is raised of whether the spherical 
approximation is always appropriate or not. The spherical approximation is reasonable for atoms having the outermost shell that is fully or half occupied since their electron densities are exactly spherical. However, in the other atoms the electron densities are not necessarily spherical, so that the conventional picture of the atomic structures is not rigorous but just an approximation. To what extent the effect of the nonspherical distribution of electrons (which is hereafter called the nonspherical effect) modifies the conventional picture of the atomic structures seems to be interesting and important. This is because the electronic structures of molecules and solids have been frequently considered on the basis of the ordinary atomic wave functions and spectra. The typical examples are the Slater integrals contained in the model Hamiltonians like the Hubbard model [10,11], and in the LDA $+U$ method $[12,13]$.

In addition to the above, there exists an obvious flaw in the spherical approximation. The total orbital angular momentum becomes necessarily zero in the conventional atomic structure, because the spherical approximation coincides with the filling approximation in which electrons are uniformly distributed into each state in the outermost shell [14]. This means that the orbital polarization never appears in the atomic structures of the spherical approximation. The orbital polarization is an origin of the magnetism of solids as well as the spin polarization [15-20], especially for the 5f-electron systems [15-17]. So far the orbital polarization has been discussed as a part of the correlation effects [17] or on the basis of the LS-multiplet theory [21]. In this paper, we shall discuss the nonspherical effect on the orbital polarization from the viewpoint of the single-particle picture. It will be shown in the following sections that the orbital polarization appears without the correlation effects.

As a first attempt to take into account the nonspherical effect, Slater has proposed a scheme for expanding the eigenfunctions of Equation (3) with the spherical harmonics [9]. However his method is difficult to be performed because an infinite number of simultaneous equations have to be solved. After Slater's proposal, there have been two kinds of approaches to this problem. One is the variational method where the single-particle wave function is expanded by using appropriately chosen basis functions $[22,23]$. Another is the density functional scheme containing the effect of the orbital current density explicitly.[24-31] In this paper, we adopt the former approach. As the basis functions, eigenfunctions for the spherical part of the single-particle potential are used and updated for each iteration of the self-consistent calculations. They are apparently different from those of the previous works $[22,23]$.

The aim of this paper is to present the tractable scheme for calculating the atomic structures beyond the spherical approximation, and to discuss the nonspherical effect. Organization of this paper is as follows. In Section 2, we present a scheme for dealing with the nonspherical effect explicitly. In order to check the validity of the scheme, we apply it to the neutral carbon atom in Section 3. The calculation procedure is also explained. The results are shown in Section 4, with a focus on the differences between the present atomic structures and the conventional one. The ground state values of the total orbital and spin angular momenta are also estimated. Finally concluding remarks are given in Section 5.

\section{A Variational Method beyond the Spherical Approximation}

In this section, we present a variational method for calculating atomic structures with taking into account the nonspherical effect.

Let us consider solving the single-particle Equation (3). The Hartree-Fock-Slater approximation is utilized [32], i.e., the effective potential of Equation (3) is given in a local form $V_{\sigma}(\boldsymbol{r})$. First, we expand the effective potential with the spherical harmonics:

$$
V_{\sigma}(\boldsymbol{r})=\sum_{l m}\left\{v_{l m}^{\sigma}(r) Y_{l m}(\theta, \varphi)+v_{l m}^{\sigma}(r)^{*} Y_{l m}(\theta, \varphi)^{*}\right\},
$$

where $v_{l m}^{\sigma}(r)$ are the radial components and their explicit forms are given in Appendix. For the convenience of the subsequent discussion, the operator of the lefthand side (LHS) of Equation (5) is defined as

$$
\hat{H}_{0}^{\sigma}:=-\frac{\mathrm{d}^{2}}{\mathrm{~d} r^{2}}+\frac{\hat{\boldsymbol{l}}^{2}}{r^{2}}+V_{\sigma}(r),
$$

where let $V_{\sigma}(r)$ be the spherically averaged potential for Equation (7), which is defined as

$$
\begin{aligned}
V_{\sigma}(r) & =\frac{1}{4 \pi} \int V_{\sigma}(\boldsymbol{r}) \sin \theta \mathrm{d} \theta \mathrm{d} \varphi \\
& =\frac{1}{\sqrt{4 \pi}}\left\{v_{00}^{\sigma}(r)+v_{00}^{\sigma}(r)^{*}\right\} .
\end{aligned}
$$

In the Expansion 7, the term $(\mathrm{Im})=(00)$ corresponds to the spherical part of the effective potential as shown in Equation (9), while the other terms correspond to the nonspherical parts.

Next, in a similar way to Equation (7), we shall expand the solution of Equation (3) with the set of known functions. As the known functions, we here adopt ones given by Equation (4), the radial part of which is the eigenfunction for the Hamiltonian 8. Thus, the solution of Equation (3) is written as

$$
\psi_{i \sigma}(\boldsymbol{r})=\frac{1}{r} \sum_{n l} \sum_{l^{\prime} m^{\prime}} C_{n l, l^{\prime} m^{\prime}}^{i \sigma} p_{n l}^{\sigma}(r) Y_{l^{\prime} m^{\prime}}(\theta, \varphi) .
$$


Substituting Equations (7) and (10) into Equation (3), and writing distinctly the spherical and nonspherical parts of the effective potential, we get

$$
\begin{aligned}
\hat{H}_{0}^{\sigma} \sum_{N} \sum_{L} C_{N L}^{i \sigma} p_{N}^{\sigma}(r) Y_{L}(\theta, \varphi)+\sum_{L^{\prime} \neq(00)}\left\{v_{L^{\prime}}^{\sigma}(r) Y_{L^{\prime}}(\theta, \varphi)\right. \\
\left.\quad+v_{L^{\prime}}^{\sigma}(r)^{*} Y_{L^{\prime}}(\theta, \varphi)^{*}\right\} \sum_{N} \sum_{L} C_{N L}^{i \sigma} p_{N}^{\sigma}(r) Y_{L}(\theta, \varphi) \\
=\varepsilon_{i \sigma} \sum_{N} \sum_{L} C_{N L}^{i \sigma} p_{N}^{\sigma}(r) Y_{L}(\theta, \varphi),
\end{aligned}
$$

where we use Equations (8) and (9), and abbreviate $n l$ as " $N$ " and $I m$ as " $L$ " for ease of seeing. It can be easily shown that Equation (11) is reduced to the spherical Equation including Equation (5), if the second term of the LHS is neglected. This means that the second term of the LHS represents the nonspherical effect that has been disregarded in the conventional spherical approximation.

Here, for simplicity, we shall use the common value of $l$ for $N$ and $L$, and suppose that the eigenvalues for the Hamiltonian 8 is denoted as $\varepsilon_{N \sigma}^{0}$. Multiplying $\frac{1}{r} p_{N_{1}}^{\sigma}(r)^{*} Y_{L_{1}}(\theta, \varphi)^{*}$ on both sides of Equation (11) and integrating over the whole space, we have

$$
\begin{aligned}
& \sum_{N} \sum_{L}\left\{\delta_{L_{1} L} O_{N_{1} N}^{\sigma}\left(\frac{\varepsilon_{N_{1} \sigma}^{0}+\varepsilon_{N \sigma}^{0}}{2}-\varepsilon_{i \sigma}\right)\right. \\
& \left.+\int p_{N_{1}}^{\sigma}(r)^{*} V_{L_{1} L}^{\sigma}(r) p_{N}^{\sigma}(r) \mathrm{d} r\right\} C_{N L}^{i \sigma}=0
\end{aligned},
$$

where

$$
O_{N_{1} N}^{\sigma}=\int p_{N_{1}}^{\sigma}(r)^{*} p_{N}^{\sigma}(r) \mathrm{d} r,
$$

$$
\begin{aligned}
& V_{L_{1} L}^{\sigma}(r) \\
& =\sum_{L^{\prime} \neq(00)} v_{L^{\prime}}^{\sigma}(r) \int Y_{L_{1}}(\theta, \varphi)^{*} Y_{L^{\prime}}(\theta, \varphi) Y_{L}(\theta, \varphi) \sin \theta \mathrm{d} \theta \mathrm{d} \varphi \\
& +\sum_{L^{\prime} \neq(00)} v_{L^{\prime}}^{\sigma}(r)^{*} \int Y_{L_{1}}(\theta, \varphi)^{*} Y_{L^{\prime}}(\theta, \varphi)^{*} Y_{L}(\theta, \varphi) \sin \theta \mathrm{d} \theta \mathrm{d} \varphi .
\end{aligned}
$$

Equation (12) is just the generalized eigenvalue problem. If the matrix elements, $O_{N_{1} N}^{\sigma}$ and $V_{N_{1} N}^{\sigma}(r)$, and the energy spectra of the spherical approximation, $\varepsilon_{N \sigma}^{0}$, are given, then we can obtain the eigenvalues, $\varepsilon_{i \sigma}$, and eigenfunctions, $\left\{C_{N L}^{i \sigma}\right\}$. It should be noted that the eigenvalues $\varepsilon_{i \sigma}$ are guaranteed to be real since both matrices of Equation (12) are hermitian. The angular integrations in Equation (14) can be analytically calculated by using the Wigner $3 \mathrm{j}$-symbols. According to the properties of the Wigner $3 j$-symbols, matrix elements of Equation (14) are zero unless $l_{1}+l^{\prime}+l=$ even , $\left|l_{1}-l\right| \leq l^{\prime} \leq l_{1}+l$ and $-m_{1}+m= \pm m^{\prime}$ [7]. These conditions also determines the upper limit of the summation of
Equation (7).

The eigenfunctions thus obtained yield the new potentials by means of the expressions given in Appendix. These potentials should coincide with the input ones. Namely, the self-consistency is required for the potentials. The corresponding basis functions in Equation (10) are modified for each iteration since the function $p_{n l}^{\sigma}(r)$ is the radial part of solution for the Hamiltonian (8) with the Potential 9. The iteration is continued until self-consistency for the potentials is achieved.

Let us show the detailed procedure of the self-consistent calculations. The flow chart of self-consistent calculations is shown in Figure 1. We first give a starting potential in some way, for example via the LDA calculation within the spherical approximation (Step 1 in Figure 1). In order to prepare the radial basis functions $p_{n l}^{\sigma}(r)$, the spherical parts of the potential are derived. Using these potentials, atomic structure calculations are performed (Step2). Then, using the basis functions and corresponding spectra, the generalized eigenvalue problem is solved (Step3). The resultant eigenfunctions provide the new potentials (Step 4). Here we check whether the potentials are converged or not (Step 5). Of course, the checking should be performed on both convergences for the spherical and nonspherical parts of potentials. If the convergence is not yet obtained, we return to Step 2 with the spherical potential calculated from the new potential. The calculations are repeated until the potentials are converged within some accuracy.

\section{Application to the Neutral Carbon Atom}

Compared to the previous ones $[9,22,23]$, the present scheme seems to be more tractable, but as to the effectiveness actual calculations have to be performed. Here we apply it to the neutral carbon atom.

In the Expansion 10, we choose the common value of $l$ in both summations for $n l$ and $l m$. That is to say, physically meaningful functions are prepared for basis functions of the expansion. In more detail, we use five functions having the following quantum numbers:

$$
(n l m)=(100),(200),(211),(210),(21-1) .
$$

Correspondingly, the upper limit of the potential given by Equation (7) is determined from the properties of the Wigner $3 \mathrm{j}$ symbols, as already mentioned below Equation (15). This time, the expansion of the potential consists of the following terms:

$$
\begin{aligned}
(\operatorname{lm})= & (00),(11),(10),(1-1),(22), \\
& (21),(20),(2-1),(2-2)
\end{aligned}
$$

The generalized eigenvalue problem is reduced to 


$$
\sum_{n l m}\left\{\delta_{l l_{1}} \delta_{m m_{1}} O_{n l n_{1} l_{1}}^{\sigma}\left(\frac{\varepsilon_{n l \sigma}^{0}+\varepsilon_{n_{1} l_{1} \sigma}^{0}}{2}-\varepsilon_{i \sigma}\right)+\int p_{n l}^{\sigma}(r)^{*} V_{l m, l_{1} m_{1}}^{\sigma}(r) p_{n_{1} l_{1}}^{\sigma}(r) \mathrm{d} r\right\} C_{n l m}^{i \sigma}=0
$$

For ease of understanding the matrices of the eigen- value problem, their explicit forms are shown below:

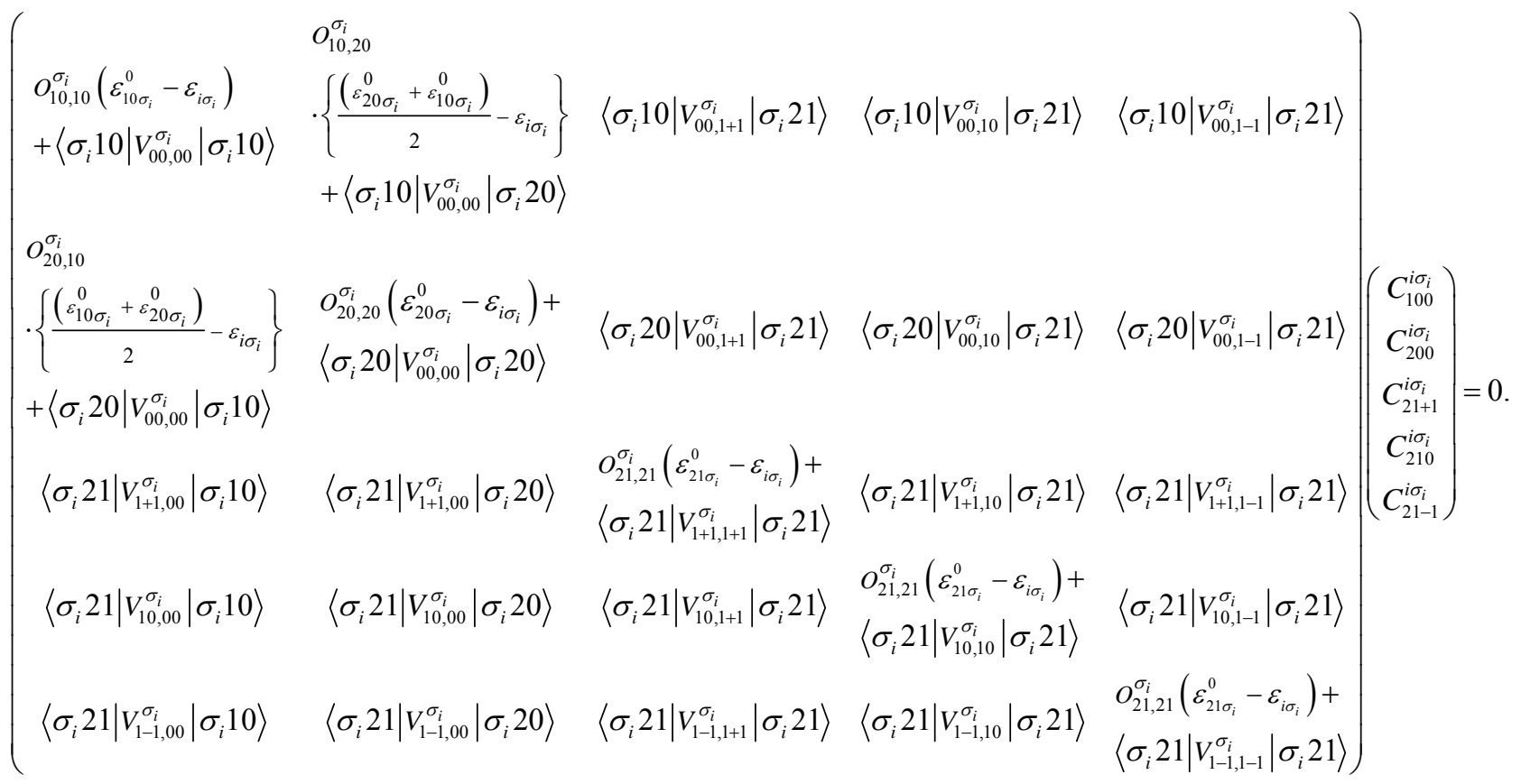

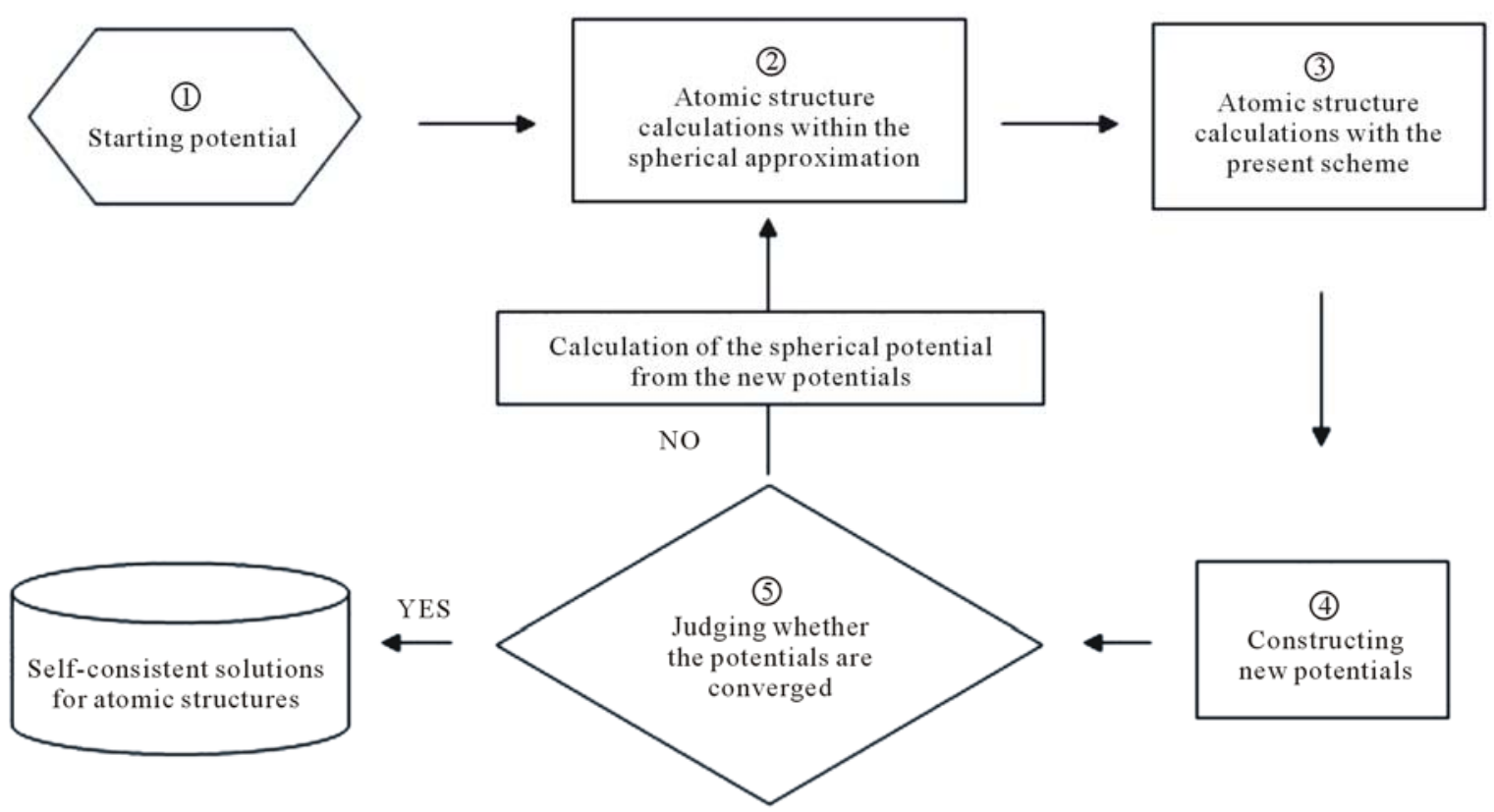

Figure 1. Flow chart of the self-consistent calculations. The detailed procedure is shown in the text.

Solving the above Equations in a self-consistent way, we can obtain the atomic structure for the neutral carbon atom. The concrete steps of the calculations are shown in the flow chart of Figure 1. Here note that there is a pos- 
sibility to exist the multiple self-consistent solutions. In order to cover such solutions, various kinds of input potentials should be arranged. This time, we prepare the orbitals of the spherical approximations, i.e. $1 \mathrm{~s}, 2 \mathrm{~s}$ and $2 p$ orbitals, and take the linear combination of them so as to construct the input potentials. The $1 \mathrm{~s}$ and $2 \mathrm{~s}$ orbitals are used as they stand, while $2 \mathrm{p}$ orbitals are transformed into the following orbitals:

$$
\begin{aligned}
\left(\begin{array}{c}
\tilde{\phi}_{1}^{\sigma} \\
\tilde{\phi}_{2}^{\sigma} \\
\tilde{\phi}_{3}^{\sigma}
\end{array}\right)= & \left(\begin{array}{ccc}
\cos \theta & \sin \theta & 0 \\
\sin \theta & -\cos \theta & 0 \\
0 & 0 & 1
\end{array}\right) \\
& \left(\begin{array}{ccc}
\cos \phi & 0 & \sin \phi \\
0 & 1 & 0 \\
\sin \phi & 0 & -\cos \phi
\end{array}\right)\left(\begin{array}{c}
\phi_{211}^{\sigma} \\
\phi_{210}^{\sigma} \\
\phi_{21-1}^{\sigma}
\end{array}\right),
\end{aligned}
$$

where $\phi_{211}^{\sigma}, \phi_{210}^{\sigma}$ and $\phi_{21-1}^{\sigma}$ are $2 \mathrm{p}$ orbitals of the spherical approximation. The input potentials are constructed from the $1 \mathrm{~s}, 2 \mathrm{~s}$ and two orbitals chosen among three ones given by Equation (17). The angles $\theta$ and $\phi$ are changed by $15^{\circ}$, respectively. A total of 864 kinds of different potentials are taken as the starting potentials $\left((360 / 15) \cdot(180 / 15) \cdot{ }_{3} C_{2}=864\right)$. As shown in a subsequent section, six kinds of self-consistent solutions can be obtained correspondingly to the starting potentials.

\section{Results and Discussions}

In this section, we will give the results of the atomic structure of the neutral carbon atom. Figure 2 shows the energy spectra of the present scheme, together with those of the conventional spherical approximation. It should be noted that the conventional atomic spectra can be specified by the quantum numbers nlm $\sigma$, but in the present scheme they are specified only by the ordinal numbers because of lack of the spherical symmetry. For instance, 1s states of the spherical approximation correspond to the 1 st and 2 nd states of the present scheme.

The conventional $2 p$ states are split into doubly degenerate levels and single one due to the nonspherical effect. There exist two types of splitting. In other words, two types of the converged self-consistent solutions (SCS) can be found from the viewpoint of the splitting of energy levels. One is that the doubly degenerate levels are higher than the single one, and another is the opposite. They are denoted as "SCS-A" and "SCS-B", respectively, in Figure 2. On the other hand, conventional 1s and $2 \mathrm{~s}$ states are little influenced by the nonspherical effect. This is because the $2 p$ states (5th and 6th states) are directly influenced by their nonspherical densities of electrons, while the wave functions for $1 \mathrm{~s}$ and $2 \mathrm{~s}$ states (from 1 st to 4 th states) are well localized near the nuclear where the spherical potential mainly caused by the nuclear is dominant.

In order to discuss the ground-state properties in more detail, we shall investigate the components of the eigenfunctions, i.e. the expansion coefficients of Equation (10). The SCS-A and SCS-B are classified into two and four types, respectively, according to the components of the
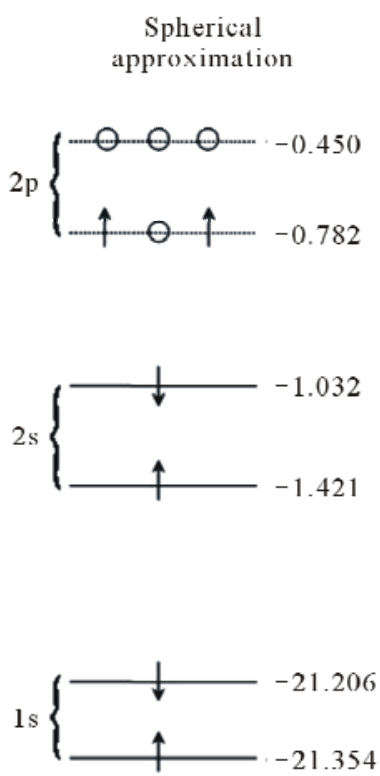
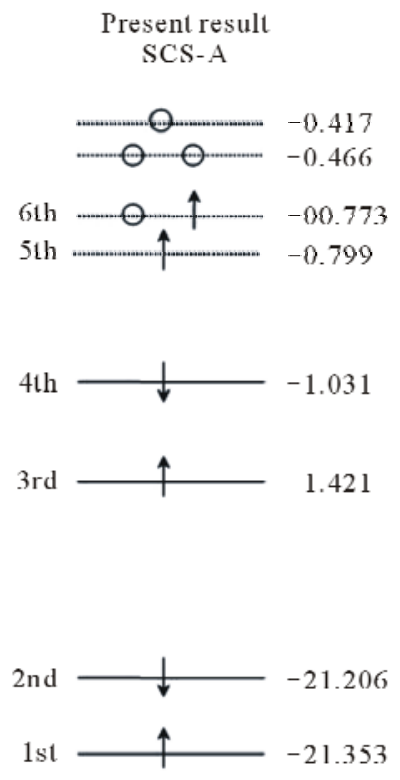
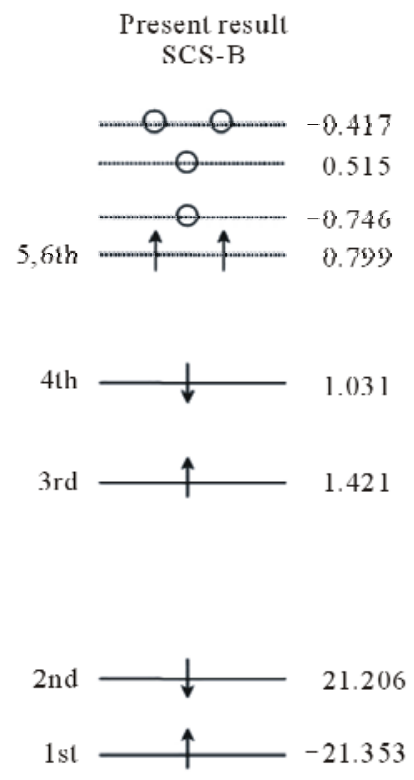

Figure 2. Energy spectra for the neutral carbon atom. The first column shows the results for the conventional spherical approximation. The second and third columns are the self-consistent solutions for the present scheme, which are denoted as "SCS-A" and "SCS-B", respectively. The up- and down-arrows denote the occupied states, and open circle the unoccupied states. All values are given in Rydberg Unit. 
wave function. They are denoted as A-1, A-2 and B-1, B-2, B-3, B-4 respectively. The expansion coefficients for the 1st, 2nd, 3rd and 4th states are shown in Table 1. For all of the coverged SCSs, the components are same as those of the spherical approximation within the accuracy of $10^{-3}$. These results are consistent with the fact that the states from 1st to 4th for the present scheme are in a good agreement with the conventional $1 \mathrm{~s}$ and $2 \mathrm{~s}$ states, respectively (Figure 1). Concerning the 5th and 6th states, there is a large difference between the present and conventional schemes, which is shown in Table 2. In the conventional spherical approximation, electrons are distributed into each shell in an equal weight, so that the corresponding coefficients are all $\sqrt{2 / 3}$. Meanwhile, components of each the converged SCS are partial to some of them. This partiality causes the polarization of the orbital angular momenta, which is so-called orbital polarization. In order to verify it, we calculate the ground state values of the total orbital and spin angular momenta. The ground state of the Hartree-Fock-Slater approximation is given by a single Slater determinant that is written as

$$
\Psi\left(x_{1}, \cdots, x_{6}\right)=\frac{1}{\sqrt{6} !}\left|\begin{array}{ccccc}
\psi_{1 \alpha}\left(\boldsymbol{r}_{1}\right) \alpha\left(\eta_{1}\right) & \cdots & \psi_{4 \alpha}\left(\boldsymbol{r}_{1}\right) \alpha\left(\eta_{1}\right) & \psi_{1 \beta}\left(\boldsymbol{r}_{1}\right) \beta\left(\eta_{1}\right) & \psi_{2 \beta}\left(\boldsymbol{r}_{1}\right) \beta\left(\eta_{1}\right) \\
\vdots & \vdots & \vdots & \vdots & \vdots \\
\psi_{1 \alpha}\left(\boldsymbol{r}_{4}\right) \alpha\left(\eta_{4}\right) & \vdots & \psi_{4 \alpha}\left(\boldsymbol{r}_{4}\right) \alpha\left(\eta_{4}\right) & \vdots & \vdots \\
\psi_{1 \alpha}\left(\boldsymbol{r}_{5}\right) \alpha\left(\eta_{5}\right) & \vdots & \vdots & \psi_{1 \beta}\left(\boldsymbol{r}_{5}\right) \beta\left(\eta_{5}\right) & \psi_{2 \beta}\left(\boldsymbol{r}_{5}\right) \beta\left(\eta_{5}\right) \\
\psi_{1 \alpha}\left(\boldsymbol{r}_{6}\right) \alpha\left(\eta_{6}\right) & \cdots & \cdots & \psi_{1 \beta}\left(\boldsymbol{r}_{6}\right) \beta\left(\eta_{6}\right) & \psi_{2 \beta}\left(\boldsymbol{r}_{6}\right) \beta\left(\eta_{6}\right)
\end{array}\right|
$$

where $x_{i}$ denote the coordinates for ith electron including spatial coordinate $\boldsymbol{r}_{i}$ and spin coordinate $\eta_{i}$, and where $\psi_{i \sigma}(\boldsymbol{r})$ is the solution of Equation (16), and where $\alpha(\eta)$ and $\beta(\eta)$ are wave functions for up- and down-spins, respectively. Using Equation (18), the total orbital angular momentum and its z-component, $L$ and $L_{z}$, are respectively calculated by

$$
\begin{aligned}
& \left\langle\Psi\left(x_{1}, \cdots x_{6}\right)\left|\hat{\boldsymbol{L}}^{2}\right| \Psi\left(x_{1}, \cdots x_{6}\right)\right\rangle=L(L+1), \\
& \left\langle\Psi\left(x_{1}, \cdots x_{6}\right)\left|\hat{L}_{z}\right| \Psi\left(x_{1}, \cdots x_{6}\right)\right\rangle=L_{z},
\end{aligned}
$$

where $\hat{\boldsymbol{L}}=\sum_{i=1}^{6} \hat{\boldsymbol{I}}_{i}$, and $\hat{\boldsymbol{I}}_{i}$ is the operator of the orbital angular momentum for the ith electron. Similarly, the total spin angular momentum and its z-component, $S$ and
$S_{z}$, are respectively given by

$$
\begin{aligned}
& \left\langle\Psi\left(x_{1}, \cdots x_{6}\right)\left|\hat{S}^{2}\right| \Psi\left(x_{1}, \cdots x_{6}\right)\right\rangle=S(S+1), \\
& \left\langle\Psi\left(x_{1}, \cdots x_{6}\right)\left|\hat{S}_{z}\right| \Psi\left(x_{1}, \cdots x_{6}\right)\right\rangle=S_{z},
\end{aligned}
$$

where $\hat{\boldsymbol{S}}=\sum_{i=1}^{6} \hat{\boldsymbol{s}}_{i}$, and $\hat{\boldsymbol{s}}_{i}$ is the operator of the spin angular momentum for the $i$ th electron. The results are shown in Table 3. In a nonrelativistic many-electron system, $L, L_{z}, S$ and $S_{z}$ are the conserved quantities. All of the coverged SCSs yield $L=1$ and $S=1$ within the accuracy of $10^{-3}$. This means that the ground states of the present scheme correspond to the term ${ }^{3} \mathrm{P}$ that is known

\begin{tabular}{|c|c|c|c|c|c|c|}
\hline & & & & $(n l m)$ & & \\
\hline \multirow{5}{*}{ Spheical approximation } & Energy [Ryd.] & $(100)$ & $(200)$ & $(21+1)$ & $(210)$ & $(21-1)$ \\
\hline & -21.354 & 1.000 & $\cdots$ & $\cdots$ & $\ldots$ & $\ldots$ \\
\hline & -21.206 & 1.000 & $\ldots$ & $\ldots$ & $\ldots$ & $\cdots$ \\
\hline & -1.421 & $\cdots$ & 1.000 & $\ldots$ & $\ldots$ & $\cdots$ \\
\hline & -1.032 & $\ldots$ & 1.000 & $\ldots$ & $\ldots$ & $\cdots$ \\
\hline \multirow{4}{*}{ Present results for SCS-A and SCS-B } & -21.353 & 1.000 & 0.000 & 0.000 & 0.000 & 0.000 \\
\hline & -21.206 & 1.000 & 0.000 & 0.000 & 0.000 & 0.000 \\
\hline & -1.421 & 0.000 & 1.000 & 0.000 & 0.000 & 0.000 \\
\hline & -1.031 & 0.000 & 1.000 & 0.000 & 0.000 & 0.000 \\
\hline
\end{tabular}
to be the ground state of the LS-multiplet theory. Furthermore, it is noticed that the present scheme obviously

Table 1. The expansion coefficients of Equation (10) for the 1st, 2nd, 3rd and 4th states. Both SCS-A and SCS-B give the same results, so we don't lable SCS-A and SCS-B distinctly. 
Table 2. The expansion coefficients of Equation (10) for the 5th and 6th states.

\begin{tabular}{|c|c|c|c|c|c|c|}
\hline & & $(n \operatorname{lm})$ & & & & \\
\hline & $\begin{array}{l}\text { Energy } \\
\text { [Ryd.] }\end{array}$ & (100) & $(200)$ & $(21+1)$ & $(210)$ & $(21-1)$ \\
\hline $\begin{array}{l}\text { Spheical } \\
\text { approximation }\end{array}$ & -0.782 & $\cdots$ & $\ldots$ & $\sqrt{2 / 3}$ & $\sqrt{2 / 3}$ & $\sqrt{2 / 3}$ \\
\hline \multirow{2}{*}{ SCS A-1 } & -0.799 & 0.000 & 0.000 & 0.000 & 1.000 & 0.000 \\
\hline & -0.773 & 0.000 & 0.000 & 1.000 & 0.000 & 0.000 \\
\hline \multirow{2}{*}{ SCS A-2 } & -0.799 & 0.000 & 0.000 & 0.000 & 1.000 & 0.000 \\
\hline & -0.733 & 0.000 & 0.000 & 0.000 & 0.000 & 1.000 \\
\hline \multirow{2}{*}{ SCS B-1 } & -0.799 & 0.000 & 0.000 & $1 / \sqrt{2}$ & 0.000 & $1 / \sqrt{2}$ \\
\hline & -0.799 & 0.000 & 0.000 & $1 / \sqrt{2}$ & 0.000 & $-1 / \sqrt{2}$ \\
\hline \multirow{2}{*}{ SCS B-2 } & -0.799 & 0.000 & 0.000 & 1.000 & 0.000 & 0.000 \\
\hline & -0.799 & 0.000 & 0.000 & 0.000 & 0.000 & 1.000 \\
\hline \multirow{2}{*}{ SCS C-1 } & -0.799 & 0.000 & 0.000 & 0.000 & 1.000 & 0.000 \\
\hline & -0.799 & 0.000 & 0.000 & $1 / \sqrt{2}$ & 0.000 & $1 / \sqrt{2}$ \\
\hline \multirow{2}{*}{ SCS C-2 } & -0.799 & 0.000 & 0.000 & 0.000 & 1.000 & 0.000 \\
\hline & -0.799 & 0.000 & 0.000 & $1 / \sqrt{2}$ & 0.000 & $-1 / \sqrt{2}$ \\
\hline
\end{tabular}

Table 3. The ground state values of the tptal orbital angular momentum and itd z-component, $L$ and $L_{Z}$, are showm in the 1st and 2nd cplumns. Also, the ground state values of the total spin angular momentum and its z-component, $S$ and $S_{Z}$, are shown in the 3rd and 4th columns.

\begin{tabular}{lllll}
\hline State & $\boldsymbol{L}$ & $\boldsymbol{L}_{Z}$ & $\boldsymbol{S}$ & $\boldsymbol{S}_{\boldsymbol{Z}}$ \\
\hline SCS A-1 & 1.000 & 1.000 & 1.000 & 1.000 \\
SCS A-2 & 1.000 & -1.000 & 1.000 & 1.000 \\
SCS B-1 & 1.000 & 0.000 & 1.000 & 1.000 \\
SCS B-2 & 1.000 & 0.000 & 1.000 & 1.000 \\
SCS B-3 & 1.000 & 0.000 & 1.000 & 1.000 \\
SCS B-4 & 1.000 & 0.000 & 1.000 & 1.000 \\
\hline
\end{tabular}

causes the orbital polarization. Since the spherical approximation never brings it, we may say that the nonspherical effect is one of the keys to the appearance of the orbital polarization.

Here note that the SCSs are classified into three kinds of states, which yield $L_{z}=1,0$ and -1 , respectively. This is not surprising because the term ${ }^{3} \mathrm{P}$ are triply degenerate with respect to the orbital angular momentum. These three states, by their nature, should be completely degenerate and their total energies should be same as each other. Actually, the total energies of these states which are also evaluated by taking the expectation values of the Hamiltonian with respect to Equation (18) coincide with each other.

\section{Concluding Remarks}

In this paper, we present a scheme for calculating the atomic structures beyond the spherical approximation and investigate to what extent the single-particle picture of atomic systems needs to be modified. It is confirmed that the orbital polarization can appear only by considering the nonspherical effect explicitly. Compared to the conventional atomic structures, we find that the atomic levels are partially split. The magnitude of splitting for $2 \mathrm{p}$ states is about $5 \%$, which is never neglected because the splitting itself causes the orbital polarization. Also, such a debacle of the conventional atomic structures seems to be conceptually important.

Although the present scheme shows the necessity of modifying the single-particle picture of atomic systems, we have to consider the following effects that are neglected in the present calculations:

1) enhancement of the expansion basis functions in Equation (10);

2) treatment of the exchange energy beyond the Hartree-Fock-Slater approximation;

3) correlation effects.

Concerning the first effect, we here adopt only $1 \mathrm{~s}, 2 \mathrm{~s}$ and $2 p$ orbitals in the expansion of the eigenfunctions. However we had better take more functions as the basis functions. Especially for the neutral carbon atom, 3d orbitals should be added to the expansion basis functions since the nondiagonal elements between the $2 p$ and $3 d$ states would not be negligibly small in Equation (12) or (15). Similarly, the second and third effects seem to be indispensable for describing the nonspherical effect in more detail. But anyway, we can say within the knowledge obtained in this paper that the orbital polarization certainly emerges by taking into account the nonspherical effect even if the correlation effects are not explicitly considered. Furthermore, the effect of the nonspherical distribution of electrons cannot be neglected not only conceptually but also quantitatively in the study on the single-particle picture of atomic systems.

\section{Acknowledgements}

This work was partially supported by Grant-in-Aid for Scientific Research (No. 19540399) and for Scientific Research in Priority Areas (No. 17064006) of The Ministry of Education, Culture, Sports, Science, and Technology, Japan. 


\section{Reference}

[1] D. R. Hartree, "The Wave Mechanics of an Atom with a Noncoulomb Central Field. PartI: Theory and Method. Part II: Some Results and Discussions," Proceedings of Cambridge Philosophical Society, Vol. 24, No. 1, 1928, 111-132.

[2] V. Fock, Z. Physik, "Näherungsmethode zur Lösung des quantenmechanischen Mehrkörperproblems," Zeitschrift Für Physik, Vol. 61, No. 1-2, pp. 126-148. doi:10.1007/BF01340294

[3] P. Hohenberg and W. Kohn, "Inhomogeneous Electron Gas," Physical Review, Vol. 136, No. 3B, 1964, pp. 864-871. doi:10.1103/PhysRev.136.B864

[4] W. Kohn and L. J. Sham, "Self-Consistent Equations Including Exchange and Correlation Effects," Physical Review, Vol. 140, No. 4A, 1965, pp. 1133-1138. doi:10.1103/PhysRev.140.A1133

[5] The spherical approximation means that the effective potential in Equation (3) is approximated into the central field, i.e., $V_{i \sigma}=V_{i \sigma}(r)$. For instance, see, J. C. Slater, "Quantum Theory of Atomic Structure," McGraw-Hill, NY, Vol. 1, 1960.

[6] This means that $V_{i \sigma}=V_{\sigma}(r)$.

[7] A. Messiah, "Quantum Mechanics", Dover Publications, NY, 1999.

[8] F. Herman and S. Skillman, "Atomic Structure Calculations," Prentice-Hall Inc., New Jersey, 1963.

[9] J. C. Slater, "The Calculation of Molecular Orbitals," John Wiley \& Sons, NY, 1979.

[10] N. F. Mott, "The Basis of the Electron Theory of Metals, with Special Reference to the Transition Metals," Proceedings of the Physical Society, London, Section A, Vol. 62, No. 7, 1949, p. 416. doi: 10.1088/0370-1298/62/7/303

[11] P. W. Anderson, "New Approach to the Theory of Superexchange Interactions," Physical Review, Vol. 115, No. 1, 1959, pp. 2-13. doi: 10.1103/PhysRev.115.2

[12] V. I. Anisimov, J. Zaanen and O. K. Andersen, "Band Theory and Mott Insulators: Hubbard $U$ instead of Stoner," Physical Review B, Vol. 44, No. 3, 1991, pp. 943-954. doi:10.1103/PhysRevB.44.943

[13] A. I. Liechtenstein, V. I. Anisimov and J. Zaanen, "Density-Functional Theory and Strong Interations: Orbital Ordering in Mott-Hubbard Insulators," Physical Review B, Vol. 52, No. 8, 1995, R5468-R5470.

[14] S. E. Koonin, "Computational Physics," Addison-Wesley, NY, 1986.

[15] O. Eriksson, B. Johansson, R. C. Albers, A. M. Boring and M. S. S. Brooks, "Orbital magnetism in Fe, Co, and Ni,” Physical Review B, Vol. 42, 1990, 2707-2710. doi:10.1103/PhysRevB.42.2707

[16] O. Eriksson, M. S. S. Brooks and B. Johansson, Phys. Rev. B 41, 7311 (1990). doi: 10.1103/PhysRevB.42.2707

[17] M. S. S. Brooks, O. Eriksson, L. Severin and B. Johansson, "Spin and Orbital Magnetization Densities in Itiner- ant Magnets" Physica B, Vol. 192, No. 1-2, 1993, pp. $39-49$.

doi:10.1016/0921-4526(93)90106-G

[18] T. Shishidou, T. Oguchi and T. Jo, "Hartree-Fock Study on the 5 f Orbital Magnetic Moment of US," Physical Review B, Vol. 59, No. 10, 1999, pp. 6813-6823. doi:10.1103/PhysRevB.59.6813

[19] M. R. Norman, "Orbital polarization and the insulating gap in the transition-metal oxides," Physical Review Letters, Vol. 64, No. 10, 1990, pp. 1162-1165. doi:10.1103/PhysRevLett.64.1162

[20] G. H. Daalderop, P. J. Kelly and M. F. H. Schuurmans, "Magnetocrystalline Anisotropy and Orbital Moments in Transition-Metal Compounds," Physical Review B, Vol. 44, No. 21, 1992, pp. 12054-12057. doi:10.1103/PhysRevB.44.12054

[21] A. Narita and M. Higuchi, "Expressions of Energy and Potential due to Orbital Polarization," Journal of the Physical Society of Japan, Vol. 75, No. 2, 2006, pp. 024301-024301-10. doi:10.1143/JPSJ.75.024301

[22] J. F. Janak and A. R. Williams, "Method for Calculating Wave Functions in a Nonspherical Potential," Physical Review B, Vol. 23, No. 12, 1981, pp. 6301-6306. doi:10.1103/PhysRevB.23.6301

[23] F. W. Kutzler and G. S. Painter, "Energies of Atoms with Nonspherical Charge Densities Calculated with Nonlocal Density-Functional Theory," Physical Review Letters, Vol. 59, No. 12, 1987, 1285-1288. doi:10.1103/PhysRevLett.59.1285

[24] A. D. Becke, "Local Exchange-Correlation Approximations and First-Row Molecular Dissociation Energies," International Journal of Quantum Chemistry, Vol. 27, No. 5, 1985, pp. 585-594. doi:10.1002/qua.560270507

[25] A. D. Becke, "Current Density in Exchange-Correlation Functionals: Application to Atomic States," Journal of Chemical Physics, Vol. 117, No. 15, 2002, pp. 6935-6938. doi: $10.1063 / 1.1503772$

[26] E. Orestes, T. Marcasso and K. Capelle, "Density-Functional Calculation of Ionization Energies of Current-Carrying Atomic States," Physical Review A, Vol. 68, No. 2, 2003, 022105. doi:10.1103/PhysRevA.68.022105

[27] E. Orestes, A. B. F. da Silva and K. Capelle, "Energy Lowering of Current-Carrying Single-Particle States in Open-Shell atoms due to an Exchange-Correlation Vector Potential," International Journal Of Quantum Chemistry, Vol. 103, No. 5, 2005, pp. 516-522. doi:10.1002/qua.20575

[28] G. Vignale and M. Rasolt, "Density-Functional Theory in Strong Magnetic Fields," Physical Review Letters, Vol. 59, No. 20, 1987, pp. 2360-2363. doi:10.1103/PhysRevLett.59.2360

[29] G. Vignale and M. Rasolt, "Current- and Spin-DensityFunctional Theory for Inhomogeneous Electronic Systems in Strong Magnetic Fields," Physical Review B, Vol. 37, No. 18, 1988, 10685-10696. doi:10.1103/PhysRevB.37.10685

[30] M. Higuchi and A. Hasegawa, "A Relativistic Current- 
and Spin-Density Functional Theory and a Single-Particle Equation," Journal of the Physical Society of Japan, Vol. 66, No. 1, 1997, p. 149 (1997). doi:10.1143/JPSJ.66.149

[31] M. Higuchi and A. Hasegawa, "Single-Particle Equation of Relativistic Current- and Spin-Density Functional Theory and Its Application to the Atomic Structure of the Lanthanide Series," Journal of the Physical Society of

\section{Appendix}

\section{Expressions for the Potentials}

In this appendix, we present the expressions for the spherical and nonspherical parts of the effective potential. The effective potential consists of three terms, i.e., the nuclear, Hartree and exchange potentials, which are given by

$$
V_{\sigma}(\boldsymbol{r})=-\frac{2 Z}{r}+2 \int \frac{\rho\left(\boldsymbol{r}^{\prime}\right)}{\left|\boldsymbol{r}-\boldsymbol{r}^{\prime}\right|} \mathrm{d} \boldsymbol{r}^{\prime}-6\left(\frac{3}{4 \pi}\right)^{\frac{1}{3}} \rho_{\sigma}(\boldsymbol{r})^{\frac{1}{3}},
$$

where the exchange potential is simplified with the aid of the Slater approximation [32], and where $\rho(\boldsymbol{r})$ and $\rho_{\sigma}(\boldsymbol{r})$ denote the electron density and electron density with $\sigma$-spin, respectively. Using Equation 10, they are written as

$$
\begin{aligned}
\rho(\boldsymbol{r}) & =\sum_{i \sigma}^{\text {occ. }}\left|\psi_{i \sigma}(\boldsymbol{r})\right|^{2} \\
& =\sum_{i \sigma}^{\text {occ. }} \frac{1}{r^{2}}\left|\sum_{n l} \sum_{l^{\prime} m^{\prime}} C_{n l, l^{\prime} m^{\prime}}^{i \sigma} p_{n l}^{\sigma}(r) Y_{l^{\prime} m^{\prime}}(\theta, \varphi)\right|^{2}, \\
\rho_{\sigma}(\boldsymbol{r}) & =\sum_{i(\sigma \text {-spin })}^{\text {occ. }}\left|\psi_{i \sigma}(\boldsymbol{r})\right|^{2} \\
& =\sum_{i(\sigma-\mathrm{spin})}^{\text {occ. }} \frac{1}{r^{2}}\left|\sum_{n l} \sum_{l^{\prime} m^{\prime}} C_{n l, l^{\prime} m^{\prime}}^{i \sigma} p_{n l}^{\sigma}(r) Y_{l^{\prime} m^{\prime}}(\theta, \varphi)\right|^{2},
\end{aligned}
$$

Japan, Vol. 67, No. 6, 1998, pp. 2037-2047. doi:10.1143/JPSJ.67.2037

[32] J. C. Slater, "A Simplification of the Hartree-Fock Method," Physical Review, Vol. 81, No. 3, 1951, pp. 385-390.doi: 10.1103/PhysRev.81.385

where the sum of Equation A3 is over only the occupied states with $\sigma$-spin. What we need are

1) spherical part of Equation A1, i.e., $V_{\sigma}(r)=$ $\frac{1}{\sqrt{4 \pi}}\left\{v_{00}^{\sigma}(r)+v_{00}^{\sigma}(r)^{*}\right\}$, that appears in Equation 8, and

2) nonspherical components of Equation A1, i.e., $v_{k q}^{\sigma}(r)$, that appear in Equation 12 or 14.

The above 1) is indispensable for deriving the basis functions $p_{n l}^{\sigma}(r)$ and corresponding spectra $\varepsilon_{n l}^{\sigma 0}$, which are also used in Equation 12 or 14.

Now let us show the explicit forms of 1) and 2) by considering each term of Equation A1. As for the first term, we have no problem because it is exactly spherical. Also, the second term can be easily separated into the spherical and nonspherical parts by means of the multipole expansion of the Coulomb potential. Concerning the third term, we have to use an approximation so as to derive one third power of $\rho_{\sigma}(\boldsymbol{r})$. Using the composition relation for the spherical harmonics, $\rho_{\sigma}(\boldsymbol{r})$ is formally separated into spherical and nonspherical parts as follows:

$$
\rho_{\sigma}(\boldsymbol{r})=\rho_{\sigma}^{S}(r)+\rho_{\sigma}^{N S}(\boldsymbol{r}),
$$

with

$$
\begin{aligned}
\rho_{\sigma}^{S}(r) & =\sum_{i(\sigma-\operatorname{spin})}^{\text {occ. }} \frac{1}{r^{2}} \sum_{n_{1} l_{1}} \sum_{l_{2} m_{2}} \sum_{n_{1}^{\prime} l_{1}} \sum_{l_{2} m_{2}^{\prime}} C_{n_{1} l_{1}, l_{2} m_{2}}^{i \sigma}{ }^{*} C_{n_{1}^{\prime} l_{1}, l_{2}^{\prime} m_{2}^{\prime}}^{i \sigma} \times p_{n_{l_{1}} l_{1}}^{\sigma}(r)^{*} p_{n_{1}^{\prime} l_{1}^{\prime}}^{\sigma}(r) \frac{(-1)^{m_{2}^{\prime}}}{8 \pi} \sqrt{\left(2 l_{2}+1\right)\left(2 l_{2}^{\prime}+1\right)} \\
& \times\left(\begin{array}{ccc}
l_{2} & l_{2}^{\prime} & 0 \\
0 & 0 & 0
\end{array}\right)\left(\begin{array}{ccc}
l_{2} & l_{2}^{\prime} & 0 \\
m_{2} & -m_{2}^{\prime} & 0
\end{array}\right)+C . C .
\end{aligned}
$$

and

$$
\begin{aligned}
& \rho_{\sigma}^{N S}(\boldsymbol{r})=\sum_{i(\sigma-\text { spin })}^{\text {ocC. }}\left|\psi_{i \sigma}(\boldsymbol{r})\right|^{2}=\sum_{i(\sigma-\text { spin })}^{\text {occ. }} \frac{1}{r^{2}} \sum_{n_{1} l_{1}} \sum_{l_{2} m_{2}} \sum_{n_{1}^{\prime} l_{1}^{\prime}} \sum_{l_{2} m_{2}^{\prime}} C_{n_{1} l_{1}, l_{2} m_{2}}^{i \sigma}{ }^{*} C_{n_{1}^{\prime} l_{1}, l_{2}^{\prime} m_{2}^{\prime}}^{i \sigma} \\
& \times p_{n_{l_{1}} l_{1}}^{\sigma}(r)^{*} p_{n_{1}^{\prime} l_{1}}^{\sigma}(r) \frac{(-1)^{m_{2}^{\prime}}}{2} \sum_{\substack{k=l_{2}-l_{2}^{\prime} \\
\neq 0}}^{l_{2}+l_{2}^{\prime}} \sum_{\substack{q=-k \\
\neq 0}}^{k}\left\{\frac{\left(2 l_{2}+1\right)\left(2 l_{2}^{\prime}+1\right)(2 k+1)}{4 \pi}\right\}^{\frac{1}{2}} \\
& \times\left(\begin{array}{ccc}
l_{2} & l_{2}^{\prime} & k \\
0 & 0 & 0
\end{array}\right)\left(\begin{array}{ccc}
l_{2} & l_{2}^{\prime} & k \\
m_{2} & -m_{2}^{\prime} & q
\end{array}\right) Y_{k q}(\theta, \varphi)+C . C . .
\end{aligned}
$$


Since $\rho_{\sigma}^{N S}(\boldsymbol{r})$ is constructed from electrons of the unfilled outermost shell alone, it is quite smaller than the spherical part of the electron density. That is to say, $\rho_{\sigma}^{S}(r) \gg \rho_{\sigma}^{N S}(\boldsymbol{r})$. Using this fact, we can get an approximate form of the one third power of $\rho_{\sigma}(\boldsymbol{r})$ as follows

$$
\rho_{\sigma}(\boldsymbol{r})^{\frac{1}{3}} \approx \rho_{S}^{\sigma}(r)^{\frac{1}{3}}+\frac{1}{3} \rho_{S}^{\sigma}(r)^{-\frac{2}{3}} \rho_{N S}^{\sigma}(\boldsymbol{r})
$$

The first and second terms turn to the spherical and nonspherical parts of the exchange potential, respectively.

Using these relations, the resultant forms of 1) and 2) are, respectively, given by

$$
\begin{aligned}
& V_{\sigma}(r)=-\frac{2 Z}{r}+\sum_{i \sigma}^{o c c} \sum_{n_{1} l_{1} l_{2} m_{2} m_{2}} \sum_{n_{1}^{\prime} l_{1}^{\prime} \sum_{2} m_{2}^{\prime}} C_{n_{l_{1}}, l_{2} m_{2}}^{i \sigma}{ }^{*} C_{n_{1}^{\prime} l_{1}^{\prime}, l_{2}^{\prime} m_{2}^{\prime}}^{i \sigma} \int \mathrm{d} r^{\prime} \frac{1}{r_{>}} p_{n_{1} l_{1}}^{\sigma}(r)^{*} p_{n_{n_{1}^{\prime} l_{1}^{\prime}}^{\sigma}}^{\sigma}(r) \\
& \times 2(-1)^{m_{2}} \sqrt{\left(2 l_{2}+1\right)\left(2 l_{2}^{\prime}+1\right)}\left(\begin{array}{ccc}
l_{2} & l_{2}^{\prime} & 0 \\
0 & 0 & 0
\end{array}\right)\left(\begin{array}{ccc}
l_{2} & l_{2}^{\prime} & 0 \\
-m_{2} & m_{2}^{\prime} & 0
\end{array}\right)-6\left(\frac{3}{4 \pi}\right)^{\frac{1}{3}} \rho_{\sigma}^{S}(r)^{\frac{1}{3}}, \\
& v_{k q}^{\sigma}(\boldsymbol{r})=\sum_{i \sigma}^{\text {occ. }} \sum_{n_{1} l_{1}} \sum_{l_{2} m_{2}} \sum_{n_{1}^{\prime} l_{1}^{\prime}} \sum_{l_{2} m_{2}^{\prime}} C_{n_{l_{1}}, l_{2} m_{2}}^{i \sigma}{ }^{*} C_{n_{1}^{\prime} l_{1}, l_{2}^{\prime} m_{2}^{\prime}}^{i \sigma} \int \mathrm{d} r^{\prime} \frac{r_{<}^{k}}{r_{>}^{k+1}} p_{n_{1} l_{1}}^{\sigma}(r)^{*} p_{n_{1}^{\prime} l_{1}}^{\sigma}(r) \\
& \times(-1)^{m_{2}^{\prime}} \sqrt{\frac{4 \pi\left(2 l_{2}+1\right)\left(2 l_{2}^{\prime}+1\right)}{2 k+1}}\left(\begin{array}{rrr}
l_{2} & l_{2}^{\prime} & k \\
0 & 0 & 0
\end{array}\right)\left(\begin{array}{ccc}
l_{2} & l_{2}^{\prime} & k \\
m_{2} & -m_{2}^{\prime} & q
\end{array}\right) \\
& -2\left(\frac{3}{4 \pi}\right)^{\frac{1}{3}} \rho_{\sigma}^{S}(r)^{-\frac{2}{3}} \sum_{i(\sigma-\text { spin })}^{\text {occ. }} \frac{1}{r^{2}} \sum_{n_{1} l_{1}} \sum_{l_{2} m_{2}} \sum_{n_{1}^{\prime} I^{\prime}} \sum_{l_{2} m_{2}^{\prime}} C_{n_{1_{1}} l_{1}, l_{2} m_{2}}^{i \sigma}{ }^{*} C_{n_{1}^{\prime} l_{2}, m_{2}^{\prime} m_{2}^{\prime}}^{i \sigma} p_{n_{1} l_{1}}^{\sigma}(r)^{*} p_{n_{1}^{\prime} l^{\prime}}^{\sigma}(r) \\
& \times \frac{(-1)^{m_{2}^{\prime}}}{2}\left\{\frac{\left(2 l_{2}+1\right)\left(2 l_{2}^{\prime}+1\right)(2 k+1)}{4 \pi}\right\}^{\frac{1}{2}}\left(\begin{array}{ccc}
l_{2} & l_{2}^{\prime} & k \\
0 & 0 & 0
\end{array}\right)\left(\begin{array}{ccc}
l_{2} & l_{2}^{\prime} & k \\
m_{2} & -m_{2}^{\prime} & q
\end{array}\right) .
\end{aligned}
$$

Here $\left(\begin{array}{ccc}l_{1} & l_{2} & l_{3} \\ m_{1} & m_{2} & m_{3}\end{array}\right)$ is the Wigner $3 j$ symbol [7], $\quad$ and $\rho_{\sigma}^{S}(r)$ is given by Equation A5. 